\title{
DA METAMATEMÁTICA PARA A CIÊNCIA COGNITIVA
}

Henrique de M orais RIBEIRO ${ }^{1}$

para o domínio da Ciência Cognitiva funcionalista neurocomputacional. A descrição de tal transição é feita por meio de uma breve análise das idéias de Post, Church, Gödel e Turing sobre a possibilidade de formalização do pensamento criador na matemática, enfatizando as contribuições deste último.

- PALAVRAS-CHAVE: Metamatemática; decidibilidade; procedimento efetivo; computabilidade; platonismo; Ciência Cognitiva.

\section{Introdução}

0 século $X X$ foi presenteado com contribuições de alto valor para 0 desenvolvimento da lógica matemática ou metamatemática. Dentre os ilustres contribuintes, podemos destacar: Kurt Gödel, Alonzo Church, Alan Mathison Turing e Émil Post. Eles depararam com questões e objetos de pesquisa semelhantes; é como se tivessem intuído os mesmos "objetos". Esses "objetos" podem ser caracterizados em forma de tópicos, a saber:

1 a capacidade de definir pura e formalmente qualquer conceito ma-

1 Mestre em Filosofia da Mente e Ciência Cognitiva. Pesquisador do Grupo Acadêmico de Estudos Cognitivos do Departamento de Filosofia da Faculdade de Filosofia e Ciências - UNESP - 17525-900 - Marília - SP - Brasil. 
temático;

2 as limitações inerentes a quaisquer sistemas formais;

3 o conceito de máquina e suas relações com o pensamento humano.

Neste trabalho, proporemos uma abordagem sobre as implicações dos trabalhos destes eminentes lógico-matemáticos para a filosofia da mente representada pelo atual ramo da Ciência Cognitiva, o funcionalismo lógico-computacional. Com semelhante abordagem, é nossa finalidade apenas mostrar alguns aspectos da ligação filosófico-histórica que há entre a lógica matemática e as pesquisas atuais na Ciência Cognitiva. Para tanto, caracterizaremos brevemente as intuições dos lógico-matemáticos; em seguida, procuraremos mostrar que o mencionado ramo dos estudos cognitivos nasceu das questões filosóficas das quais os lógicomatemáticos trataram em suas investigações.

\section{As intuições de Gödel}

Relativamente ao primeiro objeto de investigação na lógica matemática, temos as contribuições de Kurt Gödel a respeito das funções recursivas. Segundo Davis (1965, p.65), as principais contribuições de Gödel são: "G1 - Definição das funções e relações recursivas primitivas; G2 - Prova de propriedades elementares das funções recursivas". Com relação ao segundo objeto, as contribuições gödelianas foram: "G3 - Prova de que as funções e relações utilizadas para aritmetizar os sistemas formais só recursivas primitivas; G4 - Formulação do conceito de entscheindungsdefitheit (de decidibilidade)" (Davis, 1965, p.68).

Com relação ao terceiro objeto, podemos destacar: "G4 - 0 famoso teorema de Gödel e as inúmeras discussões que se seguiram com a prova do teorema". Gödel (1934) mostra, falando de forma genérica, que não é possível provar recursivamente, isto é, em termos de decidibilidade, se uma proposição de um sistema formal da aritmética elementar $\mathrm{S}$ é verdadeira ou falsa.

Gödel escolheu como sistema formal o sistema de Russel \& Whitehead (1938), demonstrando a existência de proposições formalmente indecidíveis.

Não temos espaço aqui para levantar as discussões que Gödel despertou entre os filósofos, lógicos e matemáticos. Podemos, contudo, destacar as discussões suscitadas pelo teorema que são relativas aos critérios metamatemáticos que serviram de base para o desenvolvimento da filosofia da mente. Lucas (1964) argumentou que o teorema gödeliano 
implicou conseqüências filosóficas importantes, quais sejam:

- teorema é uma legítima refutação do mecanicismo;

- pode ser interpretado como critério para distinção entre ser consciente e ser inconsciente.

- abre caminhos para conciliação entre a ética e a ciência ou entre moralidade e natureza, superando então a dicotomia kantiana entre razão pura e razão prática.

Não há espaço, neste trabalho, para avaliar os enfoques de Lucas, mas eles causaram muita repercussão filosófica. Benacerraf (1967), o principal contraditor de Lucas, mostrou que os argumentos de Lucas não são sustentáveis. Outros matemáticos, na esteira dele, defenderam teses mecanicistas apoiadas no teorema de Gödel, comparando o procedimento de formalização com a "mecanização". 0 ponto filosófico central das discussões foi então associado à questão sobre o mecanicismo: o pensamento matemático é ou não mecânico? Neste sentido, alguns lógicos matemáticos acreditavam que o teorema de Gödel implicava negação do mecanicismo; outros acreditavam que este implicava afirmação do mecanicismo. Formou-se então uma espécie, se nos é permitida a comparação, de antinomia kantiana: tese e antítese foram igualmente sustentadas.

\section{As intuições de Church}

Segundo Davis (1965), Church contribuiu com três resultados básicos: 1. A lambda definibilidade e suas conseqüências; 2 . A famosa tese de Church; 3. A noção de calculabilidade efetiva.

No que tange ao primeiro objeto, temos o cálculo lambda (the $\lambda$-calculus) criado por Church (1965) e que é uma interessante maneira de definir recursivamente conceitos matemáticos. A idéia central da definibilidade lambda é a idéia de recursividade.

É bem conhecida a tese que se seguiu das investigações de Church: a tese de Church, que pode ser situada entre as mais interessantes e controversas teses metamatemáticas. Há discussões históricas e conceituais sobre o status da tese. Se ela for pensada a partir de seu uso, pode ser assim enunciada: Toda função efetivamente calculável é recursiva geral. A noção de procedimento efetivo (effetiveness), inerente à tese, é bastante discutida e o próprio Church parecia não ter uma idéia clara sobre esta noção. Nelson (1987, p.581) afirma que a discussão ainda pros- 
segue: "embora a tese de Church tenha sido importante para a teoria da decidibilidade efetiva durante cinqüenta anos, a questão que envolve seu status epistemológico ainda permanece aberta".

Por status epistemológico, entende-se, a nosso ver, a questão de se a referida tese é ou não uma questão empírica - sua veracidade depende da experiência - ou pura - sua veracidade depende apenas da prova de novos teoremas da lógica matemática. No primeiro caso, é interessante notar que ela remete a questões filosóficas sobre a natureza da atividade cognitivo-criativa humana em matemática, que pertenceriam ao domín io da Psicologia cognitiva Empírica.

0 terceiro objeto está intimamente relacionado com o segundo: as relações entre a noção de efetividade e a compreensão da atividade cognitiva na elaboração criativa da matemática; para Nelson (1987, p.581):

A interpretação empiricista da TC pode ser sugerida pelos argumentos a favor de uma teoria mecanicista da mente, teoria esta que a tese de Church apóia. Os argumentos podem ser resumidos da forma seguinte: Os processos cognitivos humanos são efetivos.

"Efetividade" no fragmento acima é comparado a "procedimento mecânico", mencionado junto ao trabalho de Gödel.

\section{As intuições de Turing}

Alan Mathison Turing não poderia não fazer parte do grupo de revolucionários. Independentemente dos trabalhos de Church e Gödel, Turing [1939] apresentou uma noção equivalente à noção de recursividade geral intuída por Church e Gödel, e também intuiu outra forma do primeiro "objeto". Trata-se da definabilidade de conceitos matemáticos por computabilidade, e esta seria a noção essencial. Turing (1939, p.119) primeiramente aplica sua definição de números: “Um número é computável se ele difere de um inteiro se comparado com um número computado por uma máquina livre de círculos".

Não vamos discutir aqui o que Turing entende por máquina livre de círculos, mas o que nos interessa é mostrar que ele procurava uma nova forma de definir rigorosa e formalmente as noções matemáticas intuitivas. Nesse caso, notamos que faz apelo à noção de máquina e suas relações com o pensamento, e com isso intuiu o terceiro "objeto", qual seja: a máquina de Turing.

A forma como Turing analisou o conceito de calculabilidade efetiva 
se dá na situação de um computador humano que realiza cálculos. Os elementos que ele considerou relevantes para caracterizar seus "estados mentais" são:

1 meio é uma fita potencialmente infinita dividida em quadrados;

2 número de quadrados imprimíveis é finito;

3 número de quadrados observados é finito;

4 número de estados mentais do computador é finito;

5 as operações do computador constituem uma ordem discreta;

6 as operações são basicamente a troca de símbolo de um dos quadrados observados junto com uma possível troca do estado mental, e a troca de um dos quadrados observados por outro (o qual está no máximo a uma certa distância fixa dos quadrados previamente observados) junto com uma possível troca do estado mental.

É importante salientar que Turing faz uma ressalva: "Todos os argumentos que apresento estão fundamentalmente atrelados a apelos à intuição e, por essa razão, serão mais insatisfatórios do ponto de vista matemático" (Davis, 1965, p.135).

Essa ressalva pressupõe a imaginação quando trabalha com a "máquina de papel" proposta por Turing. Seu artefato matemático provocou posteriormente bastante reação no meio filosófico e o próprio Turing aprofundou consideravelmente suas intuições. Nos anos posteriores ao trabalho de 1936, poderíamos dizer que sua imaginação ganhou progressivamente mais proporções; a intuição do terceiro objeto - a relação entre o conceito de máquina e o pensamento - é descrita por Shanker (1987, p.626) da forma seguinte:

A transição que se pode notar no pensamento de Turing está intimamente ligada com a transformação gradual dos seus interesses, que partem da teoria da recursão para a Inteligência Artificial. Dado o espírito empreendedor que se encontrava na teoria da recursão - para mostrar que todas as funções da teoria dos números são recursivamente calculáveis - , Turing, imbuído desse espírito, sentiu-se obrigado a mostrar que todas as funções efetivas da teoria dos números eram mecanicamente calculáveis. Em conseqüência disso, ele planejou demonstrar que seu computador mecânico seria tão poderoso quanto o computador matemático, isso é, "humano".

Turing (1950) reformula suas concepções de modo a defender completamente a tese mecanicista na filosofia da mente, contendo o já conhecido argumento do "jogo da imitação" (the imitation game). A situação, entretanto, foi invertida, pois não se trata de limitar a atividade de um ser humano, isto é, representar o humano computador, mas de repre- 
sentar o computador humano, ou seja, de se explorar a possibilidade de uma máquina pensar ou de apresentar inteligência humana. Essa pretensão básica de Turing definiu os pródromos de uma futura ciência, a Ciência Cognitiva, que comentaremos na segunda parte do trabalho.

\section{As intuições de Post}

Com relação ao primeiro objeto, Post teve suas intuições reunidas em seu sistema de conjuntos geráveis (generable sets), os quais são uma interessante maneira de definir noções matemáticas. Os conjuntos geráveis formam o que Post chamava sistemas de produção (produtions systems), dentre os sistemas canônicos (canonical systems) eram os mais importantes. Segundo Cutland (1980):

1 um alfabeto finito, isto é, um conjunto finito de símbolos;

2 um subconjunto do conjunto de todas as combinações possíveis do alfabeto, ou seja, o conjunto de palavras, denominado axiomas;

3 um conjunto básico de produções, ou de regras de transformação de palavras em outras palavras.

Post utilizou os sistemas de produção para geração de conjuntos a fim de caracterizar o sistema formal, o cálculo proposicional de Russel \& Whitehead (1938). Caracterizou tal sistema formal como um sistema canônico de produções transformadoras de axiomas em teoremas.

Com essa caracterização, Post intuiu o segundo "objeto": as limitações do pensamento formalizador, quando demonstrou a incompletude do sistema canônico formulado segundo o cálculo proposicional de Russel $\&$ Whitehead, em moldes semelhantes aos que foram definidos por Gödel treze anos antes (cf. Davis, 1965, p.381).

Quando Post chegou ao resultado de incompletude do Principia, passou, cremos, a intuir o terceiro objeto, isto é, começou a pensar no conceito de procedimento mecânico, chegando até, antes de Turing, a propor um conceito de máquina. Segundo Davis (1965), a máquina de Post pode ser definida como se segue:

1 uma fita infinita nos dois sentidos, dividida em quadrados que podem estar vazios ou conter uma marca, dos quais seleciona-se um como ponto inicial;

2 um conjunto de ordens baseadas nas seguintes operações:

2.1 marcar o quadrado atual;

2.2 apagar o quadrado atual; 
2.3 mover o quadrado para a direita;

2.4 mover o quadrado para a esquerda;

2.5 determinar se o quadrado atual está ou não marcado.

Seqüência de passos semelhantes aos que foram definidos por Turing. Partindo dessa intuição, Post passou a se interessar por uma investigação empírica sobre a natureza da mente, isto é, das condições do pensamento criador matemático, como podemos notar no fragmento abaixo:

Estabelecer esta universalidade não é questão de prova matemática, mas de análise psicológica dos processos mentais envolvidos nos processos matemáticos combinatórios ... (Davis, 1965, p.418)

Em outro trecho afirma-se: " $M$ as para a generalidade total, teria que ser feita uma análise completa de todas as possíveis maneiras em que a mente humana pudesse iniciar os processos finitos para gerar seqüências" (Davis, 1965, p.408)

Post referia-se à generalidade e à universalidade de certos processos formais, sobre os quais ele acreditava ser necessário um estudo empírico da mente humana. Quando provou a incompletude do sistema canônico de Russel \& Whitehead, procurou posteriormente iniciar uma pesquisa empírica sobre a natureza dos estados mentais. Para Post, a tese de Church não seria resolvida matematicamente, mas empiricamente; enveredou, então, por caminhos empíricos para investigar as limitações do poder formalizador, indo contra o posterior mecanicismo de Turing. Como observa Oddifreddi (1989, p.105):

[Post] propôs a identificação das noções de conjunto de cadeias finitas efetivamente geráveis, por um lado, e geráveis por sistemas canônicos, por outro. [lsto é] indi retamente equivalente à tese de Church (para as funções recursivas parciais). Porém, Post tinha uma visão filosófica platônica, e considerou sua proposta como algo que tinha que ser provado de alguma maneira, por uma espécie de "análise psicológica dos processos mentais envolvidos nos processos matemáticos combinatórios".

Post, a nosso ver, intuíra o primeiro "objeto" e com isso antecipara direções modernas de pesquisa da Ciência Cognitiva.

Consideraremos a seguir o estudo do que podemos denominar elo platônico entre os trabalhos dos lógicos matemáticos.

\section{0 platonismo em matemática²}


Cantor foi um platonista, um crente em uma realidade matemática que transcende a mente humana. Estas construções e estes mundos imaginários impõem-nos, então, a sua ordem. Temos que reconhecer sua objetividade. (Davis \& Rersch, 1987)

As fortes "intuições de objetos" que aventamos, a nosso ver, podem constituir premissas de um argumento histórico-filosófico para a filosofia platonista da matemática, ou visão realista sobre os processos matemáticos. Acreditamos que os lógicos matemáticos não apenas formalizaram ou construíram novos "nomes" ou "definições", mas outrossim intuíram realidades matemáticas.

Nossa crença é apoiada por um resultado metamatemático segundo o qual os conceitos, relativamente aos três objetos intuídos por Turing, Church, Gödel e Post, são equivalentes. Tal prova de equivalência constitui outra premissa importante, do ponto de vista formal, de um argumento platonista.

Gödel, por exemplo, mostra sua concepção platonista no trecho seguinte:

Malg rado seu distanciamento da experiência dos sentidos, temos algo como que uma percepção também dos objetos da teoria dos conjuntos, como se depreende do fato de que os axiomas se impõem a nós como verdadeiros. Não vejo nenhuma razão por que deveríamos ter menos confiança neste tipo de percepção, isto é, na intuição matemática, do que na percepção dos sentidos. Esses objetos podem também representar um aspecto da realidade objetiva. (Davis $\&$ Rersch,1987, p.360)

Por "aspectos de uma realidade objetiva" representada por "objetos" podemos entender, relativamente ao resultados da lógica matemática que discutimos anteriormente (cabe a teoria dos conjuntos), aqueles três objetos anteriormente descritos.

Paralelamente ao platonismo que parece embasar as intuições dos grandes lógicos matemáticos, parece haver uma certa intuição de que a realidade matemática é passível de uma abordagem empírica. Davis \& Rersch (1987, p.359) confirma semelhante possibilidade, ao definir o platonismo na filosofia da matemática: "Segundo o platonismo, um matemático é uma cientista empírico, como um geólogo: não pode inventar

2 Agradecemos, em especial, ao professor Cosme Damião Bastos Massi, que durante aulas de lógica matemática, inspirou-nos esta seção de nosso trabalho. 
nada, pois tudo já existe. 0 que se pode fazer é descobrir coisas".

É sobre esse tipo empírico de intuição, que liga a lógica matemática com a filosofia da mente e, posteriormente, com a nova Ciência Cognitiva, que falaremos a seguir.

\section{Filosofia da mente e as três intuições}

Já abordamos as intuições dos metamatemáticos relativamente ao conceito de máquina e suas relações com o pensamento. Quando eles se propuseram a pensar sobre tal conceito, passaram também a pensar sobre certos domínios do que hoje podemos chamar de filosofia da mente que, geralmente falando, se dedica à investigação filosófica sobre a natureza de nossos estados mentais. Como vimos, eles cogitaram sobre a questão de se saber se o próprio processo de pensamento humano seria ou não formalizável.

No caso de Gödel, constatamos as interessantes (e, até hoje, intermináveis) discussões sobre se seu teorema de limitação dos sistemas formais é ou não uma prova contra a tese de que nosso pensamento seja realizado de forma puramente mecânica. No caso de Post (cf., por exemplo, Davis 1965), podem-se investigar suas conjecturas sobre a natureza dos processos psicológicos, que considerava não-mecânicos, envolvidos no processo psicológico-cognitivo de manipulação de símbolos. A nosso ver, tanto Post quanto Gödel enveredaram para uma investigação profunda sobre a natureza não-mecânica - criadora - do pensamento matemático.

Turing, entretanto, foi diferente: passou da metamatemática para 0 que se pode chamar hoje de Ciência Cognitiva, relativamente à corrente funcionalista lógico-computacional ou Inteligência A rtificial (IA). Os jovens fundadores da IA, Allen Newell, Herbert Simon, Marvin Minsky e J ohn MacCarthy, herdaram os ideais de Turing, dados os objetivos de um dos encontros catalíticos para a formação da Ciência Cognitiva, o Simpósio de Hixon. ${ }^{3}$ Esses ideais têm sua origem nos principais trabaIhos de Turing como notamos no fragmento seguinte:

Mesmo em seu maior trabalho de pós-guerra (Proposal for Development in the Mathematics Division of an Automatic Computing Engine, escrito em 1946, e o London Lecture, escrito em 1947), ele estava ainda prioritariamente tratando da mecanização dos procedimentos matemáticos; isto é, do problema da determinação do "escopo da máquina", o qual ele naturalmente enfocou por meio de

30 leitor interessado pode consultar, por exemplo, Gardner (1995), capítulo sexto da parte I. 
Esse desejo de mecanização progressiva do pensamento matemático (e, depois, do pensamento em geral) inspirou as pesquisas de Turing na área de IA . Ainda para Shanker (1987, p.627):

Turing achou no jogo de xadrez a essência da infra-estrutura que fundamentava os "números computáveis" dos recursos para construção da superestrutura mecanicista que ele quis erigir por meio daquela fundamentação. 0 xadrez representou 0 salto categorial que partiu da força bruta dos algoritmos automodificadores, pavimentando o caminho para uma mudança abrupta do aspecto inicial das idéias do artigo On Computable Numbers, no qual não se exigia que as máquinas estivessem prontas para aprender, a partir de jogadas anteriores, a aprimorar a qualidade da nova jogada. Essa prontidão foi 0 argumento mais recente que o levou a introduzir a noção de máquina inteligente, no ano seguinte, na London Lecture, em que ele defendeu, de forma explícita, sua tese mecanicista.

0 jogo de xadrez foi o que Gardner (1995) chamou de "a ferramenta do especialista" (the expert tool) dos futuros teóricos da inteligência artificial. 0 trecho a seguir é longo, mas ilustra nosso argumento a respeito da influência de Turing sobre Gardner (p.153):

No verão de 1956, um grupo de dez jovens acadêmicos com formação em matemática e lógica reuniu-se no campus do Dartmouth, em Hanover, New Hampshire. Seu propósito: discutir as possibilidades de se produzir programas computacionais que pudessem "se comportar" ou "pensar" de maneira inteligente. Como eles haviam declarado em sua solicitação de bolsa para a Fundação Rockefeller: "O estudo será conduzido com base na hipótese de que todo aspecto da aprendizagem ou de qualquer outra faceta da inteligência pode, em princípio, ser descrito de forma tão precisa que se pode fazer que uma máquina o simule".

As hipóteses de Turing, relativas às semelhanças entre uma máquina e o homem que se poderiam determinar, no contexto da IA, se converteram em hipóteses empíricas. Porque as simulações computacionais são essenciais, na IA, para uma prog ressiva mecanização ou prog ramação de determinada atividade cognitiva que se queira investigar, tendo 0 computador como recurso empírico para se testar uma determinada hipótese sobre tal atividade. Na seção a seguir, definiremos algumas conclusões que detalham os aspectos empíricos da Ciência Cognitiva.

\section{Conclusões: 0 empirismo da Ciência Cognitiva}


0 desenvolvimento que os criadores da IA realizaram com base nas idéias de Turing, a nosso ver, justifica em parte a tradição empirista que se realizou, em sua máxima expressão, nos modernos estudos da Ciência Cognitiva. Porque a tese de Turing, na Inteligência Artificial, se converteu em uma hipótese empírica, como vimos. As últimas preocupações de Post, interpretadas como platonistas, eram puramente empíricas do ponto de vista da pesquisa da natureza dos estados mentais, isto é, da lei natural que rege os estados psicológicos associados com a possibilidade de um sistema de produção, da própria natureza da matematização realizada pelo matemático (cf. Davis,1965, p.291 s.).

Por outro lado, o platonismo de Gödel coloca seu teorema como uma porta que, se aberta, nos conduz a uma forma empírica de refutar o mecanicismo. Argumentos filosóficos para semelhante refutação foram elaborados por Putnam (1989).

A tese de Church foi recentemente interpretada como uma hipótese empírica favorável ao mecanicismo, como podemos notar em Nelson (1987, p.581):

Embora a tese de Church tenha sido central para a teoria da decidibilidade efetiva durante cinqüenta anos, seu status epistemológico ainda é uma questão aberta. Do meu ponto de vista, o que pode ser afirmado, partindo-se de uma posição naturalista perante questões matemáticas, é que a tese é uma proposição empírica da Ciência Cognitiva, que está aberta a confirmações, para ser aceita ou descartada, o que aparentemente parece ser verdadeiro. 0 naturalismo, embora não sendo reconhecido como uma escola da filosofia da matemática, tal como o historicismo de Webb, é algo intensamente novo: esta escola remonta até Quine que insistia que a epistemologia da matemática deve ser, em princípio, um ramo da psicologia. Neste artigo, eu desejo somente defender a metatese limitada de que a tese de Church é empírica, além de matemática.

A posição de Nelson reflete e justifica o que pode ser chamado "virada empirista da matemática" na Ciência Cognitiva. Não por acaso, Gardner (1995, p.19) define, num escopo maior, Ciência Cognitiva da forma seguinte:

Defino Ciência Cognitiva como um esforço contemporâneo para, com fundamentação empírica, resolver questões de longa data, principalmente aquelas relativas à natureza de questões epistemológicas de longa data, suas origens, seu desenvolvimento e seu emprego.

A Epistemologia, pela definição de Gardner, é tomada num sentido bem mais amplo. Obviamente, ela deve cobrir a epistemologia da matemática. As intuições dos g randes metamatemáticos, que deram origem à 
IA, contribuíram bastante para o aparecimento da Ciência Cognitiva. As questões metamatemáticas sobre a natureza da atividade matematizante dos matemáticos foram incorporadas no enfoque naturalista-cognitivista do sujeito do conhecimento matemático. Temos, assim, uma interessante transição, ou melhor, uma metamorphosis por meio da qual as controvérsias teóricas entre lógicos matemáticos se transformam em um programa de pesquisa claramente definido e, atualmente, com pleno desenvolvimento nas chamadas Ciências da Computação, a Inteligência A rtificial.

RIBEIRO, H. de M. From metamathematics to Cognitive Science. Trans/Form/ Ação (São Paulo), v.21-22, p.181-93, 1998-1999.

- ABSTRACT: In this article, it is suggested a possible profile for the historical and philosophical migration of several issues from the metamathematical domain to the domain of functionalist neuro-computational Cognitive Science. The description of such a transition is accomplished by an analysis of the ideas of Post, Church, Gödel, and, in particular, Turing on the possibility of formalization of creative thinking in Mathematics.

- KEYWORDS: Metamathematics; decidability; effective procedure; computability; Platonism; Cognitive Science.

\section{Referências bibliográficas}

BENACERRAF, P. God, Evil and Gödel. Monist, v.51, p.9-32, 1967.

$\mathrm{CHURCH}, \mathrm{A}$. An unsolvable problem of elementar number theory. In: DAVIS, M. The Undecidable. New York: Cambridge University Press, 1965.

CUTLAND, N. Computability: an introduction to recursive function theory. New York: Cambridge University Press, 1980.

DALE, J. Metamathematical criteria for minds and machines. Erkenntnis, v.27, p.1-16, 1987.

DAVIS, M. The Undecidable. New York: Cambridge University Press, 1965.

DAVIS, J. P., RERSCH, R. A experiência matemática. Trad. J. B. Pitombeira. Rio de Janeiro: Francisco Alves, 1987. 
GARDNER, H. A nova ciência da mente: uma história da revolução cognitiva. São Paulo: Edusp, 1995.

LUCAS, J. R. Minds, machines and Gödel. In: ANDERSON, A. R. Minds and machines. New Jersey: Prentice Hall, 1964.

NELSON, R. J. Church thesis and cognitive science. The Notre Dame J ournal of Formal Logic, v.28, n.4, p.581-613, 1987.

ODDIFREDDI, P. Classical Recursion Theory. Amsterdam: North-Holland, 1989.

PUTNAM, H. Minds and machines. In: Realism and Reason. Cambridge: Cambridge University Press, 1983. (Philosophical Papers, 3v). . Representation and reality. Cambridge: M IT Press, 1989.

RUSSELL, B., WHITEHEAD, A. N. Principia mathematica. Cambridge: Cambridge University Press, 1938. v.1

SHANKER, G. S. Wittgenstein versus Turing on the nature of Church's Thesis. The Notre Dame J ournal of Formal Logic, v.28, n.4, p.615-49, 1987.

TURING, A. M. Systems of Logic Based on Ordinals. In: Proccedings of the London Mathematical Society, v.2, 1939.

. Computer machinery and intelligence. Mind, v.59, p.433-60, 1950.

. A. M. Systems of Logic based on ordinals. In: DAVIS, M. The Undecidable. New York: Cambridge University Press, 1965.

NIETZSCHE, F. Cinco prefácios para cinco livros não escritos. Trad. Pedro Süssekind. São Paulo: Sette Letras, 1996. 